\title{
Ensamble estacional de aves marinas en la pesquería del palangre artesanal de la merluza austral Merluccius australis, en canales subantárticos de Chile
}

Seasonal seabird assemblages in the artisanal long-line fishery of austral hake Merluccius australis, in Chile's sub-Antarctic channels

\author{
Jaime Ojeda ${ }^{1,2,3}$, Cristián G. $\operatorname{Suazo}^{4}$ y Jaime R. Rau ${ }^{5}$
}

\author{
${ }^{1}$ Departamento de Ciencias y Recursos Naturales, Facultad de Ciencias, Universidad de Magallanes, Casilla 113-D, Punta \\ Arenas, Chile \\ ${ }^{2}$ Parque Etnobotánico Omora, Universidad de Magallanes, Teniente Muñoz 396, Puerto Williams, Chile \\ ${ }^{3}$ Instituto de Ecología y Biodiversidad, Casilla 653, Santiago, Chile. jaimeojedavillarroel@yahoo.es \\ ${ }^{4}$ Instituto de Ecología y Evolución, Facultad de Ciencias, Universidad Austral de Chile, Casilla 567, Valdivia, Chile \\ ${ }^{5}$ Laboratorio de Ecología, Departamento de Ciencias Básicas \& Programa IBAM, Universidad de Los Lagos, Casilla 933, \\ Osorno, Chile
}

\begin{abstract}
The fjords and channels of southern Chile represent breeding and feeding areas for sub-Antarctic seabird species. In addition, in these environments, an important artisanal austral hake Merluccius australis longline fishery operates. Studies on the relationship between seabirds and this activity are fundamental for the ecology and conservation biology of these birds. This study examined the composition and structure of the seasonal seabird assemblages attending the artisanal longline fishing activities in channels of the Southeast Pacific, Chile. Fieldwork was carried-out during summer (Cook Bay), winter (Felix Lighthouse), and spring (Gulf Xaultegua) of 2008. Ten species were associated with fishing activities. The most common seabird species observed during the year were the Black-browed albatross Thalassarche melanophrys and the Southern giant-petrel Macronectes giganteus. The seasonal structure of the assemblages presented differences between summer and winter. In terms of abundance, Black-browed albatrosses presented the highest contribution to the assemblage in summer (87.5\%). During winter the Kelp gull Larus dominicanus showed the highest contribution (47.7\%) while the Black-browed albatross abundance decreased to $9.1 \%$. Black-browed albatross was the species with the highest index of relative importance within the assemblies, but is also a species with the conservation status 'endangered'. Therefore, it is necessary to maintain constant monitoring of the interaction between seabirds and artisanal longline fishing activities of sub-Antarctic environments.
\end{abstract}

Key words: Black-browed albatross, Southeast Pacific, artisanal fishery, demersal fishery, species richness

Resumen.- Los fiordos y canales subantárticos de Chile presentan importantes áreas de alimentación y reproducción de aves marinas. Además, estos ambientes albergan una importante actividad de pesca de palangre artesanal de la merluza austral (Merluccius australis). Los estudios de asociaciones entre aves marinas y la actividad pesquera son fundamentales en la ecología y biología de la conservación de estas aves. Por esta razón, se investigó la composición y estructura del ensamble estacional de aves marinas relacionado con actividades de pesca de palangre artesanal de merluza austral en canales del Pacífico sudeste, Chile. El trabajo de campo fue llevado a cabo durante verano (Bahía Cook), invierno (Faro Félix) y primavera (Golfo Xaultegua) del año 2008. Diez especies fueron asociadas con actividades artesanales, las más comunes durante el año fueron el albatros de ceja negra Thalassarche melanophrys y el petrel gigante antártico Macronectes giganteus. La estructura estacional del ensamble presentó diferencias entre verano e invierno. En términos de abundancia, el albatros de ceja negra mostró la más alta contribución al ensamble en verano (87,5\%). Durante invierno, la gaviota dominicana Larus dominicanus, presentó la más alta contribución (47,7\%), mientras que el albatros de ceja negra disminuyó a 9,1\%. El albatros de ceja negra fue la especie con mayor Índice de Importancia Relativa dentro del ensamble, pero también es una especie con estatus de conservación 'en peligro', por lo tanto es necesario mantener un monitoreo constante sobre la interacción entre las aves marinas y la pesca del palangre artesanal en estos ambientes subantárticos.

Palabras clave: Albatros de ceja negra, Pacífico sudeste, pesca artesanal, pesquería demersal, riqueza de especies 


\section{INTRODUCCIÓN}

La pesquería con palangre es el método más utilizado en todos los océanos y mares del mundo (Tasker et al. 2000) y ha sido implementado en la pesquería demersal de carácter industrial y artesanal. De esta actividad, las mayores concentraciones de extracción a nivel mundial provienen de las capturas del bacalao, halibuts y merluza (Tasker et al. 2000). Este método pesquero genera un foco de atracción permanente para las aves marinas, facilitando tanto interacciones negativas (e.g., mortalidad por anzuelos), como positivas (e.g., aumento de la oferta de carroña) (Montevecchi 2002).

Dentro del sistema de canales y fiordos subantárticos del sur de Chile ( $\left.41^{\circ} 20^{\prime}-55^{\circ} 58^{\prime} \mathrm{S}\right)$, la pesquería con palangre es desarrollada exclusivamente por la pesquería artesanal. La principal especie extraída es la merluza austral Merluccius australis (Hutton, 1872), la cual fue la especie más capturada por la pesquería artesanal del palangre, durante el año 2008, en la región de Magallanes y Antártica Chilena con 2.008 ton (SERNAPESCA 200811). En cuanto a los estudios sobre la asociación entre aves marinas y la pesquería artesanal del palangre en la zona de canales subantárticos, existen escasos estudios, uno de los aportes más notables ha sido realizado por Moreno et al. (2006), donde se han destacado seis especies asociadas: albatros de ceja negra Thalassarche melanophrys (Temminck, 1828), petrel negro Procellaria aequinoctialis (Linnaeus, 1758), petrel gigante Macronectes sp., gaviota dominicana Larus dominicanus (Lichtenstein, 1823), petrel plateado Fulmarus glacialoides (Smith, 1840) y pingüino Spheniscus sp., destacándose la gaviota dominicana por presentar las mayores abundancias durante otoño, invierno y verano (Moreno et al. 2006).

El esfuerzo de captura de la merluza austral en la pesquería del palangre artesanal de los canales subantárticos ha aumentado: durante el año 1999 se utilizaron 900.000 anzuelos duplicándose para el año 2002 (Moreno et al. 2006). Sin embargo, esta pesquería es considerada de bajo impacto negativo para las aves debido a la baja tasa de captura incidental por anzuelo (0,03 aves cada 1000 anzuelos) (Moreno et al. 2006). Para la pesquería del palangre industrial de merluza este tipo de información es incierta, ya que no existen datos disponibles de mortalidad incidental de aves marinas para la región oceánica del sur de Chile (Anderson et al. 2011). No obstante, los artes de pesca del palangre artesanal se han adaptado a las actividades industriales, con el fin de reducir significativamente la mortalidad de aves marinas (Moreno et al. 2008). El caso más significativo se representa por la pesquería del bacalao de profundidad (Dissostichus eleginoides, Smitt 1898), donde la adaptación de los artes de pesca de la flota artesanal a la industrial han traído como consecuencia una disminución notable en la mortalidad de aves marinas con un promedio de 1.542 aves muertas para el año 2002 versus cero el año 2006 (Moreno et al. 2008).

El ecosistema de canales y fiordos subantárticos ha alcanzado un importante rol en la conservación de las aves marinas, debido principalmente a tres razones: i) la región de Magallanes en gran parte incluye una zona de endemismo de aves marinas para Chile (e.g., zona subantártica), donde al menos 15 especies pertenecientes al componente subantártico poseen colonias reproductivas (Schlatter \& Simeone 1999); ii) en esta zona se encuentra aproximadamente el $20 \%$ de la población mundial de albatros de ceja negra, con al menos 6 sitios de nidificación (Moreno \& Robertson 2008), considerada como una especie globalmente en peligro (BirdLife International 20102); iii) en la zona de canales y fiordos de la región de Magallanes, se estima que se encuentran cerca de un millón de individuos de pingüino de Magallanes, siendo la isla Magdalena el centro de nidificación más importante de Chile (Venegas 1999, Bingham \& Hermann 2008).

A pesar de la baja tasa de captura incidental de aves marinas por la pesquería del palangre artesanal de los canales subantárticos chilenos (Moreno et al. 2006), existen potenciales efectos aún no evaluados para esta región. Se ha planteado que las pesquerías podrían mantener poblaciones de aves marinas en tamaños artificiales debido al descarte de peces y eviscerados de las actividades de pesca industrial (Arata \& Xavier 2003). Con respecto a la pesca artesanal, si bien cada embarcación tiene un menor esfuerzo pesquero que un buque industrial, el gran número de embarcaciones artesanales que operan en conjunto, probablemente lleguen a ser parte significativa del total del esfuerzo de pesca (Moreno et al. 2006) y es razonable suponer que suceda lo mismo con el descarte y desecho de vísceras, aun cuando esto no ha sido evaluado.

${ }^{1}$ SERNAPESCA. 2008. Estadística pesquera del desembarque artesanal del año 2008 para Chile. Servicio Nacional de Pesca, Valparaíso. [en línea] <http: / / www.sernapesca.cl>

${ }^{2}$ BirdLife International. 2010. Ficha descriptiva para Thalassarche melanophrys [en línea] <http://www.birdlife.org> 
Cualquier cambio futuro que pueda experimentar el esfuerzo pesquero artesanal, podría desencadenar respuestas demográficas aún desconocidas para las aves vinculadas a esta actividad productiva. Sólo mediante la identificación del ensamble de aves que interactúa con la pesca artesanal y el monitoreo de sus interacciones, será posible la conservación de la biodiversidad en este complejo ambiente marino. Sobre la base de lo expuesto, el objetivo del presente trabajo fue evaluar la composición, riqueza de especies y la abundancia estacional de aves marinas asociadas a la pesquería artesanal de merluza austral en la zona de canales subantárticos del sur de Chile.

\section{Materiales y MÉTOdos}

\section{ÁREA DE ESTUDIO Y DESCRIPCIÓN DE LA PESQUERÍA}

El estudio se desarrolló durante el año 2008 en los canales subantárticos del sur Chile, específicamente en la región de Magallanes y Antártica Chilena. Los sitios de estudio

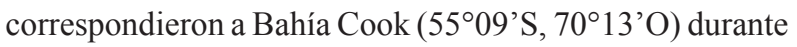
febrero (verano austral), Faro Félix (52 $\left.54^{\circ} \mathrm{S}, 7^{\circ} 03^{\prime} \mathrm{O}\right)$ durante julio (invierno austral) y Golfo Xaultegua ( $53^{\circ} 05^{\prime} \mathrm{S}$, $73^{\circ} 21^{\prime} \mathrm{O}$ ) durante octubre (primavera austral) (Fig. 1). Esta región se encuentra dominada por el viento oeste, alcanzando velocidades superiores a $90 \mathrm{~km} \mathrm{~h}^{-1}$ (Santana et al. 2006), junto a un patrón espacial variable de precipitaciones que puede alcanzar valores superiores a $4.500 \mathrm{~mm}$ anuales (Aravena \& Luckman 2009). Estos canales poseen una masa de agua superficial de origen subantártico, la que a medida que ingresa al interior de los fiordos es mezclada con agua dulce proveniente de glaciares y ríos (Silva \& Calvete 2002).

La flota pesquera artesanal de merluza austral está constituida principalmente por embarcaciones entre 7 a $10 \mathrm{~m}$ de eslora, con motores de 45 HP y compuesta generalmente por dos pescadores. El arte de pesca es desarrollado en dos modalidades, espineles móviles conocidos como 'atorrantes' y espineles fijos denominados 'línea madre' (Moreno et al. 2006). Las faenas de extracción se prolongan durante 1 a 3 meses por zona de pesca. El establecimiento de la flota depende de la biomasa extraída, además de las condiciones climáticas que permitan el operar de la pesquería. Así, las faenas se desplazan por el interior de los canales a lo largo de un año. Es importante destacar que existe un receso de esta pesquería durante el mes de agosto, debido a la veda biológica reproductiva de merluza austral en Chile (Subsecretaria de Pesca 201133).

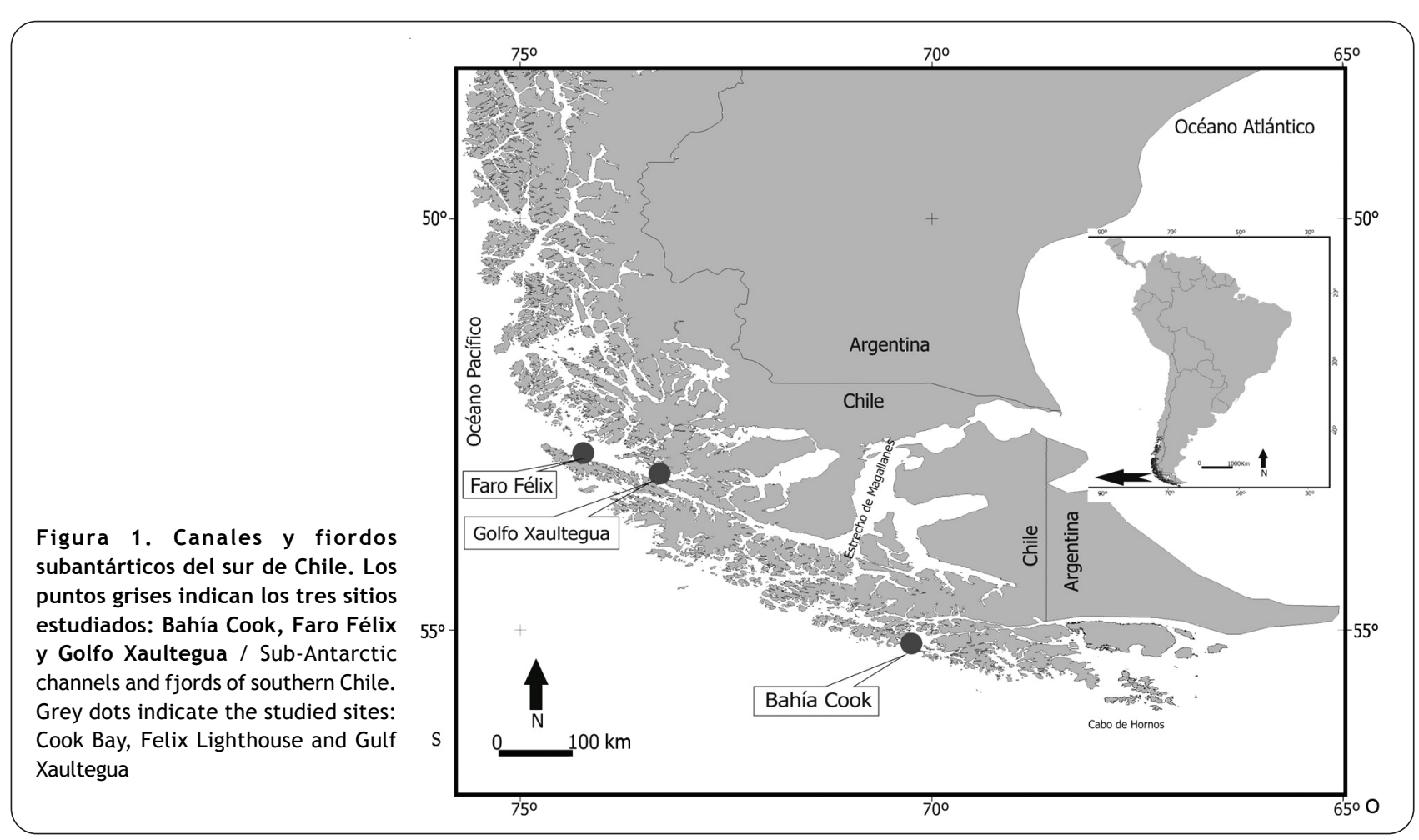

${ }^{3}$ Subsecretaria de Pesca, Gobierno de Chile. 2011. Decreto exento 140, artículo 1: Establece la veda biológica para la merluza austral (Merluccius australis). [en línea] <http://www.subpesca.cl> 


\section{OBTENCIÓN Y ANÁLISIS DE DATOS}

La composición, riqueza y abundancia de aves marinas se estimó mediante un punto fijo de conteo (Bibby et al. 2000) cubriendo un arco de $360^{\circ}$ de visibilidad a una distancia límite de 100 a 150 m desde la embarcación durante 10 min (Tasker et al. 1984). Este se efectuó durante el virado del material de pesca (entre las 14:00 y 18:00 h) y específicamente cuando se realizaba el despojo de los eviscerados de merluza austral, debido a que: i) Hudson \& Furness (1988) observaron que la evisceración de los peces es el proceso que produce mayor abundancia de aves dentro del operar de pesca, y ii) mediante observaciones personales en terreno, se detectó que el ensamble de aves en estudio se concentraba en las cercanías de la embarcación, lo que favorece el reconocimiento de especies y el conteo de individuos. Por lo tanto, estas consideraciones fueron tomadas para aumentar la detección de las especies de aves asociadas a la embarcación y no sobreestimar la abundancia de aves por conteos dobles de individuos. Los registros se obtuvieron durante 17 días distribuidos en diferentes estaciones del año: ocho en verano, cinco en invierno y cuatro en primavera.

La riqueza de especies fue expresada como el número de especies identificadas asociadas a las faenas de pesca y la abundancia de individuos por especie fue expresada en individuos contabilizados por bote y por día (ind ${ }^{-1}$ bote $^{-1}$ día $\left.^{-1}\right)$. Para estimar la riqueza esperada se generaron curvas de rarefacción para cada estación (ver Moreno 2001, García \& Goméz-Laich 2007), utilizando como referencia el valor de abundancia de la muestra de menor tamaño. Para detectar teóricamente si nuestro esfuerzo de muestreo durante el año logró estimar el total de las especies asociadas a la pesquería, utilizamos los modelos de acumulación de especies de Clench y de dependencia lineal, ambos fueron diseñados para la estimación de la riqueza de especies dependiendo del número de muestras (Soberón \& Llorente 1993). Para la estimación de los coeficientes de cada modelo de regresión no lineal, se utilizaron el método de estimación Simplex y Quasi-Newton del paquete estadístico STATISTICA 7 (StatSoft 1999²).

Para determinar los cambios estacionales del ensamble de aves (i.e., entre verano, invierno y primavera), se realizó un escalamiento multidimensional MDS, basado en la matriz de distancia ecológica de Bray-Curtis (Clarke \& Gorley 2001). Para docimar diferencias estadísticas entre las estaciones del año, se utilizó el análisis de similitud
ANOSIM (Clarke \& Warwick 2001). Esta prueba cuando entrega un valor del estadígrafo R cercano a cero sugiere que las diferencias entre los grupos son mínimas, y cuando éste es cercano a uno sugiere que las diferencias entre los grupos son máximas (Beuchel \& Gulliksen 2008). También se estimó el índice de diversidad alfa de Simpson $(\lambda)=\Sigma \mathrm{p}_{i}^{2}$, donde $\mathrm{p}_{i}=$ es la abundancia proporcional de la especie $i$, es decir, el número de individuos de la especie $i$ dividido entre el número total de individuos de la muestra (Simpson 1949).

Para la caracterización del ensamble por especie, se determinaron parámetros e índices comunitarios tales como: media, error estándar, frecuencia de ocurrencia relativa (número de muestras en que cada especie estuvo presente con respecto al total de los censos realizados), y el porcentaje de contribución SIMPER. Este último fue estimado mediante la abundancia de individuos por muestra, permitiendo medir la contribución de cada especie con la disimilitud promedio de Bray-Curtis entre grupos de muestras (Clarke \& Gorley 2001). Se utilizó un Índice de Importancia Relativa (IIR) como estimador general de la importancia de cada especie en el área (ver Cursach et al. 2010a), según la expresión IIR $=100(\mathrm{Ni} / \mathrm{Nt})$ ( $\mathrm{M} i / \mathrm{Mt}$ ), donde $\mathrm{N} i$ es la suma de las abundancias estacionales de la especie $i$ durante todo el período de estudio, Nt es el valor de la abundancia total de aves durante todo el período de estudio, $\mathrm{M} i$ es el número de conteos en los que la especie $i$ estuvo presente y Mt es el número total de conteos. Los análisis estadísticos de la estructura estacional del ensamble fueron realizados utilizando el paquete estadístico PRIMER 5.2 (Clarke \& Gorley 2001).

\section{Resultados}

Durante todo el periodo de estudio las especies de aves marinas asociadas a la pesquería artesanal de merluza austral fueron en total de diez: albatros de ceja negra, petrel negro, petrel gigante antártico Macronectes giganteus (Gmelin, 1789), petrel damero Daption capense (Linnaeus, 1758), petrel plateado, fardela negra Puffinus griseus (Gmelin, 1789), cormorán imperial Phalacrocorax atriceps (King, 1828), pingüino de Magallanes Spheniscus magellanicus (Forster, 1781), skua común Stercorarius chilensis (Bonaparte, 1857) y gaviota dominicana. Primavera fue la estación con mayor riqueza de especies observada y esperada con 7 y 6,6 especies respectivamente. Las especies con mayor frecuencia de ocurrencia relativa durante todo el periodo de

${ }^{4}$ StatSoft. 1999. STATISTICA for Windows [Computer Program Manual], Tulsa. [en línea] <http://www.statsoft.com> 
estudio, fueron el petrel gigante antártico y el albatros de ceja negra con un $100 \%$ de ocurrencia durante todas las estaciones. Tambien el albatros de ceja negra fue la especie con el mayor Índice de Importancia Relativa (IIR=47,4) (Tabla 1).

La predicción estimada para la riqueza de especies asociadas al esfuerzo de muestreo determinada mediante el modelo de Clench, mostró que los valores de las constantes fueron $\mathrm{a}=1,506 \mathrm{y} \mathrm{b}=0,078$. La relación de estos valores $(\mathrm{a} / \mathrm{b})$ arrojó una riqueza máxima esperable de 19,2 especies (valor de la asíntota de la curva de acumulación de especies con un $\mathrm{R}^{2}=0,93$ ). En cuanto, al modelo de dependencia lineal, las constantes fueron $\mathrm{a}=$ 1,402 y $b=0,109$; por lo tanto la riqueza máxima esperable (a/b), fue de 12,8 especies con un $\mathrm{R}^{2}=0,93$, valor cercano al observado empíricamente en el presente estudio. Por lo tanto, los dos modelos presentados sugieren aumentar el esfuerzo de muestreo para alcanzar la asíntota en la estimación de especies asociadas a la pesquería artesanal de merluza austral.

Los resultados del análisis MDS (stress $=0,06$ ) muestran una clara distancia estacional del ensamble de aves marinas entre el periodo estival (primavera-verano) y el periodo invernal (Fig. 2). La prueba estadística ANOSIM determinó diferencias significativas entre la estación de verano e invierno $(R=1 ; P<0,05)$. Esto confirma estadísticamente la distancia observada con el análisis MDS. A su vez, el índice de Simpson muestra que el verano es la estación que presenta mayor diversidad de especies y el invierno es la estación con menor diversidad (Tabla 1).

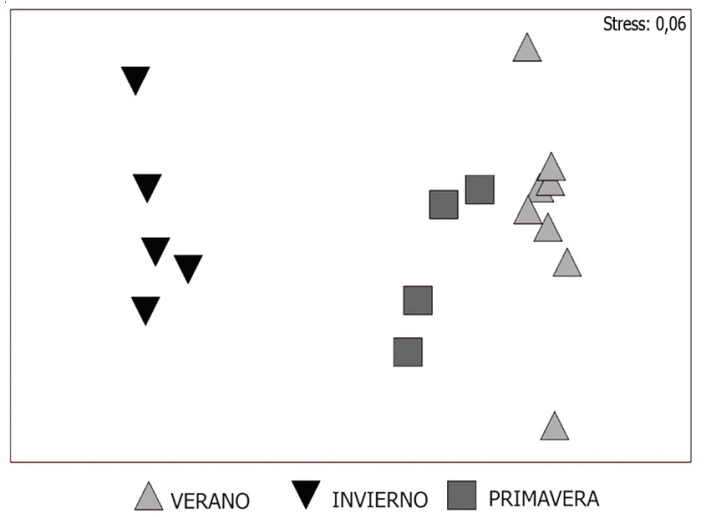

Figura 2. Escalamiento Multidimensional (MDS) para la abundancia estacional de especies de aves marinas asociadas a la pesquería artesanal de la merluza austral. Los datos no fueron transformados y se usó la matriz de distancia ecológica de BrayCurtis / Multi-dimensional Scaling (MDS) for seabird seasonal abundance associated with the artisanal austral hake fishery. Data was not transformed and the ecological distance matrix of BrayCurtis was applied

Tabla 1. Media, error estándar ( \pm E.E), abundancia total estacional por especie, frecuencia de ocurrencia relativa (FR), Índice de Importancia Relativa (IIR) y porcentaje de contribución SIMPER (\%C) de las aves marinas asociadas a la pesquería artesanal de merluza austral en canales subantárticos de Chile. Diversidad alfa del ensamble estacional mediante la riqueza observada, riqueza esperada y el índice de Simpson $(\lambda)$. $N^{\circ}$ ind = número de individuos / Mean, standard error ( \pm E.E), total seasonal abundance by species, relative frequency of occurrence (FR), index of relative importance (IIR), and SIMPER percent contribution (\%C) of seabirds associated with the artisanal fisheries of austral hake in sub-Antarctic channels of Chile. Alpha diversity of seasonal assemblage through observed richness, expected richness and Simpson index $(\lambda)$. $N^{\circ}$ ind $=$ numbers of individuals

\begin{tabular}{|c|c|c|c|c|c|c|c|c|c|c|c|}
\hline \multirow[b]{2}{*}{ Especies } & \multicolumn{3}{|c|}{ Verano } & \multicolumn{3}{|c|}{ Invierno } & \multicolumn{3}{|c|}{ Primavera } & \multirow[b]{2}{*}{ FR } & \multirow[b]{2}{*}{ IIR } \\
\hline & Media \pm E.E & $\mathrm{N}^{\circ}$ ind & $\% \mathrm{C}$ & Media \pm E.E & $\mathrm{N}^{\circ}$ ind & $\% \mathrm{C}$ & Media \pm E.E & $\mathrm{N}^{\circ}$ ind & $\% \mathrm{C}$ & & \\
\hline T. melanophrys & $41,3 \pm 4$ & 330 & 87,6 & $5 \pm 1,3$ & 25 & 9,1 & $54,8 \pm 13,2$ & 219 & 54,6 & 100,0 & 47,4 \\
\hline P. aequinoctialis & - & 2 & - & $0,6 \pm 0,2$ & 3 & 0,7 & $5 \pm 2,4$ & 20 & 2,2 & 41,2 & 0,9 \\
\hline M. giganteus & $5,3 \pm 1,8$ & 42 & 6,3 & $9,6 \pm 2,5$ & 48 & 13,3 & $19 \pm 5,5$ & 76 & 16,3 & 100,0 & 13,7 \\
\hline D. capense & - & 0 & - & $3,4 \pm 0,9$ & 17 & 6,6 & - & 1 & - & 29,4 & 0,4 \\
\hline F. glacialoides & - & 0 & - & $10,8 \pm 4,5$ & 54 & 22,5 & $1,5 \pm 0,6$ & 6 & 1,2 & 47,1 & 2,3 \\
\hline P. griseus & - & 90 & - & - & 0 & - & - & 0 & - & 5,9 & 0,4 \\
\hline P. atriceps & - & 2 & - & - & 0 & - & - & 0 & - & 5,9 & 0 \\
\hline S. magellanicus & - & 4 & - & - & 0 & - & - & 0 & - & 5,9 & 0 \\
\hline S. chilensis & $4,9 \pm 1,7$ & 39 & 6,2 & - & 0 & - & $15,5 \pm 2,5$ & 62 & 17,4 & 64,7 & 5,4 \\
\hline L. dominicanus & - & 0 & - & $24 \pm 4,5$ & 120 & 47,8 & $12,5 \pm 4,6$ & 50 & 8,3 & 52,9 & 7,4 \\
\hline $\mathrm{N}^{0}$ individuos totales & \multicolumn{3}{|c|}{509} & \multicolumn{3}{|c|}{267} & \multicolumn{3}{|c|}{434} & & \\
\hline Riqueza observada & \multicolumn{3}{|c|}{7} & \multicolumn{3}{|c|}{6} & \multicolumn{3}{|c|}{7} & & \\
\hline Riqueza esperada & \multicolumn{3}{|c|}{6,4} & \multicolumn{3}{|c|}{6} & \multicolumn{3}{|c|}{6,6} & & \\
\hline Diversidad de Simpson $(\lambda)$ & \multicolumn{3}{|c|}{0,46} & \multicolumn{3}{|c|}{0,28} & \multicolumn{3}{|c|}{0,32} & & \\
\hline
\end{tabular}


La estación de verano presentó el mayor número total de individuos por estación (509 individuos). Particularmente, durante esta estación el albatros de ceja negra presentó la mayor abundancia dentro del ensamble de aves (Tabla 1). También se destacó la presencia ocasional de especies como el cormorán imperial, la fardela negra y el pingüino de Magallanes. Éstas, fueron definidas como especies ocasionales (i.e., de baja frecuencia), ya que se encontraron presentes sólo durante un censo en el periodo de estudio.

La estación de invierno registró el menor número total de individuos por estación (267 individuos). En cuanto a la estructura del ensamble, se caracterizó por la mayor abundancia de la gaviota dominicana y una disminución en la abundancia del albatros de ceja negra (Tabla 1). También se observó la contribución de aves de origen antártico como el petrel damero y el petrel plateado, esta última especie contribuyó al 22,5\% del ensamble de aves para invierno (Tabla 1).

En primavera se observó nuevamente un aumento del número total de individuos por estación (434 individuos). En cuanto a la estructura del ensamble, se detectó un aumento en la abundancia del albatros de ceja negra y una disminución en la abundancia de la gaviota dominicana (Tabla 1). La contribución al ensamble del petrel plateado disminuyó a un $1,2 \%$ en esta estación.

\section{Discusión}

Estudios recientes efectuados en el norte del sistema de canales subantárticos de Chile, han descrito principalmente solo a 6 especies de aves asociadas a la pesquería bajo estudio (Moreno et al. 2006). Sin embargo, en el presente trabajo se aumentaron los registros a 10 especies asociadas a este tipo de pesquería, valor que es similar al predicho por el modelo de dependencia lineal (12,2 especies), el cual es adecuado para regiones amplias (véase Moreno 2001). Otras especies no registradas en nuestro estudio, pero que se han observado durante la primavera del 2010 en faenas de pesca artesanal de merluza austral en el estrecho de Magallanes (J. Ojeda, obs. pers.), son el petrel azul Halobaena caerulea (Gmelin, 1789) y el petrel de Nueva Zelanda Procellaria westlandica (Falla, 1946). Además, censos ornitológicos han proporcionado datos que muestran que en la zona del archipiélago subantártico (canal Messier), el petrel de Nueva Zelanda constituye más del $80 \%$ de los individuos que pertenecen al género Procellaria (Fraser 2009). Estimamos, entonces, en 12 el número potencial de especies de aves marinas que podrían asociarse a futuro a esta pesquería.
La variabilidad estacional en la riqueza y abundancia de especies en el ensamble podría estar relacionada a los movimientos migratorios. Por ejemplo, en el caso del petrel plateado y del petrel damero estuvieron ausentes en verano y presentes en invierno y primavera, con una abundancia mayor en invierno. Su presencia más representativa en abundancia dentro del ensamble invernal, estaría dada por la migración durante esta estación desde sus colonias reproductivas ubicadas en el arco de Escocia y la península Antártica (Weidinger 1997, Creuwels et al. 2007), hacia aguas subantárticas y subtropicales (Jehl 1973, Creuwels et al. 2007). Ambas especies han sido observadas solo en la estación invernal en estudios previos, por ejemplo Jehl (1973) durante invierno, registró a lo largo de la costa chilena al petrel plateado en la cercanías de Valparaíso ( $33^{\circ} 40^{\prime} \mathrm{S}, 74^{\circ} 00^{\prime} \mathrm{O}$ ) $\mathrm{y}$ al petrel damero en las proximidades de la isla San Félix $\left(29^{\circ} \mathrm{S}\right)$. En cambio Brown et al. (1975), mencionaron la ausencia de estas dos especies durante el periodo estival (i.e., marzo) para el estrecho de Magallanes.

Especies tales como el cormorán imperial, la fardela negra y el pingüino de Magallanes fueron observadas sólo una vez a lo largo del periodo de estudio. Si bien su asociación con la pesca artesanal de merluza austral es de baja frecuencia, estas especies corresponden a las más conspicuas del sistema de canales y fiordos. De hecho, el cormorán imperial en Chile presenta el mayor número de sitios de nidificación en la región de Magallanes (Cursach et al. 2010b) y es una de las aves marinas más abundantes en el sistema de canales y fiordos (Imberti 2005). La baja detección de estas aves en el campo, probablemente esté atribuida a sus hábitos de alimentación, e.g., el cormorán imperial y el pingüino de Magallanes obtienen sus presas por medio de buceos (Orta 1992, Johnsgard 1993, Williams 1995) dificultando su observación. Además, el reducido número de ejemplares de ambas especies registrado en este estudio podría estar relacionado con su dieta, que consiste principalmente de peces pelágicos como las sardinas (Ferrari et al. 2004, Wilson et al. 2005) y no de merluza. Sin embargo, tradicionalmente en la pesca del palangre los cebos de los anzuelos son sardinas, correspondiendo a un foco potencial de atracción de alimentación y de muerte incidental para estas aves buceadoras. Lo anterior, concuerda con las observaciones efectuadas en el área de estudio donde se registró la muerte por anzuelo de un individuo de pingüino de Magallanes, evento también observado en el archipiélago de Los Chonos $\left(46^{\circ} \mathrm{S}\right)$ donde se ha registrado este tipo de muerte incidental (Suazo datos no publicados). 
Este estudio realizado en la zona sur de los canales subantárticos, detectó que la mayor contribución en abundancia durante las estaciones de verano, invierno y primavera fue compartida por dos especies: mientras que la gaviota dominicana aumentó su abundancia en el periodo invernal, el albatros de ceja negra lo hizo en el estival (primavera-verano). Moreno et al. (2006) en la zona norte de los canales subantárticos (i.e., entre $42^{\circ}$ y $48^{\circ} \mathrm{S}$ ), identificaron a la gaviota dominicana como la especie más abundante durante las estaciones de otoño, invierno y verano. Esta diferencia de dominancia en términos de abundancia de especies en el periodo estival entre la zona norte y sur de los canales subantárticos (gaviota dominicana y albatros de ceja negra, respectivamente), probablemente esté asociada a la gran cantidad de colonias reproductivas de albatros de ceja negra emplazadas en las cercanías de la zona sur (ver Moreno \& Roberston 2008).

El aumento de la abundancia de albatros de ceja negra en el periodo estival concuerda con el ciclo reproductivo de esta especie, que comienza entre septiembre-octubre y finaliza en mayo (Tickell \& Pinder 1975). El desplazamiento del albatros de ceja negra en el periodo reproductivo es variable, puede presentar desplazamientos amplios hacia el Frente Polar Antártico o circundar las aguas subantárticas (Phillips et al. 2008). Sin embargo, dentro del periodo reproductivo, específicamente en la fase de guarda, la amplitud de desplazamiento disminuye, restringiéndose casi exclusivamente alrededor de las colonias (Phillips et al. 2008). Durante el periodo no reproductivo el desplazamiento y la distribución se amplía nuevamente, con respecto a las colonias reproductivas (e.g., Mackley et al. 2010). Para la zona de las islas Diego Ramírez se ha planteado que, durante el periodo reproductivo, los adultos se desplazan hacia la plataforma continental de Chile y Argentina (Arata \& Xavier 2003). Esta asociación ha sido propuesta debido a que los albatros de ceja negra consumen especies provenientes de esta zona, tales como los crustáceos Munida spp. y el cefalópodo Loligo gahi (Orbigny, 1835) (Arata \& Xavier 2003).

Alrededor y al interior de la región sur del archipiélago subantártico se han descrito 6 colonias reproductivas de albatros de ceja negra, correspondiendo al $20 \%$ de la población mundial (Moreno \& Roberston 2008). Por lo tanto, la relación trófica de estos albatros con el interior del archipiélago subantártico puede constituir un importante rol en la dinámica poblacional de esta especie, siendo reconocido el uso de fiordos y canales por estas aves (Suazo 2008). Esta relación se reafirma por al menos tres razones: i) el descubrimiento relativamente reciente de numerosas colonias reproductivas del albatros de ceja negra al interior y alrededor del sistema de fiordos y canales subantárticos (e.g., Aguayo et al. 2003); ii) la presencia de Munida spp., uno de los decápodos más importantes en términos de abundancia en el sistema de canales y fiordos (Arntz et al. 1999), siendo un ítem presa importante en la dieta de los albatros de ceja negra que nidifican en las islas Diego Ramírez (Arata \& Xavier 2003); iii) la oferta de descarte de la pesca artesanal de merluza austral, principalmente constituido por la merluza de cola Macruronus magellanicus (Lönnberg, 1907) y las estructuras blandas como las vísceras de merluza austral (e.g., hígados, gónadas y estómagos), siendo los hígados el ítem preferencial en el consumo de carroña de estas aves (Ojeda \& Suazo 20085).

El albatros de ceja negra es un ave de mediano tamaño corporal, con una envergadura alar media de 2,16 m (Pennycuick 1982) y una biomasa media de $3,9 \mathrm{~kg}$ (Tickell 2000). Dentro de los canales, este albatros y el petrel gigante antártico son las aves de mayor envergadura alar y biomasa que componen el ensamble asociado a la pesca artesanal de merluza austral. Esta condición no ocurre en la zona oceánica subantártica, donde el ensamble de aves asociado a la pesquería del palangre artesanal e industrial y a la pesquería del arrastre industrial, se encuentra conformado por especies considerablemente de mayor tamaño y biomasa corporal, tales como el albatros errante Diomedea exulans (Linnaeus, 1758) y el albatros real del sur Diomedea epomophora (Lesson, 1825) (Moreno et al. 1996, Weimerskirch et al. 2000, Moreno et al. 2006, González-Zevallos \& Yorio 2006). Probablemente esta segregación en la distribución de especies entre zonas abiertas y al interior de canales, posiciona al albatros de ceja negra, como un carroñero tope en el consumo de descarte pesquero en el archipiélago subantártico.

Cabe destacar que la pesquería artesanal puede ser reconocida como una actividad de bajo impacto negativo

\footnotetext{
${ }^{5}$ Ojeda J \& C Suazo. 2008. Aves marinas y desechos de la pesquería artesanal de la meluza austral (Merluccius australis) en canales sur-patagónicos de Chile. Boletín Chileno de Ornitología (Chile), volumen 14 (Número extraordinario, Resúmenes IX congreso chileno de ornitología). [en línea] <http:// www.unorch.cl>
} 
hacia las aves marinas, al compararse con actividades de tipo industrial. No obstante, a raíz de su significativo esfuerzo pesquero en aguas chilenas (ver Moreno et al. 2006), también se presenta como un importante modelo para representar las interacciones con la biota asociada a dicha actividad. De esta forma, el presente trabajo proporciona antecedentes que demuestran la fuerte interacción y dependencia de consumidores marinos ante el subsidio de alimento desde la pesquería demersal artesanal, más allá del periodo reproductivo. La caracterización de estos ensambles de aves y su relación con la pesquería, adquiere una gran importancia al considerar como fuertes componentes de éstos, a especies en peligro de extinción a nivel global como el albatros de ceja negra. Además, actualmente para este tipo de especies existen vacíos acerca de su distribución y actividad post-reproductiva, sobre todo desde las importantes colonias del Pacífico sudeste, tales como las islas subantárticas chilenas (Wakefield et al. 2011). Por lo tanto, los estudios estacionales del ensamble de aves asociado a la pesquería artesanal, tanto descriptivos como experimentales, explorando las interacciones positivas como negativas de esta asociación, permitirán potenciar una mayor información acerca de las poblaciones amenazadas de petreles y albatros del sur de Sudamérica.

\section{Agradecimientos}

A los pescadores artesanales de merluza austral de la región de Magallanes en especial a Sr. Enrique Vera y Pablo Román por compartir su experiencia sobre pesquería artesanal austral. A Sr. José Pérez por su colaboración en terreno. Al Dr. Andrés Mansilla, Paula Caballero y a los dos evaluadores anónimos por su apoyo y comentarios al término del presente manuscrito. J. Ojeda agradece al programa de Magíster en Ciencias con Mención en Manejo y Conservación de Recursos Subantárticos de la Universidad de Magallanes y a la beca de Magíster del Proyecto ICM, código P05-002 otorgada por el IEB. C.G. Suazo agradece el apoyo de CONICYT. Finalmente, se agradece al Pacific Seabird Group (Craig S. Harrison Conservation Grant) y al Instituto de Fomento Pesquero (Sede Punta Arenas) por todo el apoyo brindado.

\section{LiTERATURA CITADA}

Aguayo A, J Acevedo \& P Acuña. 2003. Nuevo sitio de anidamiento del albatros ceja negra, Diomedea melanophris Temmink 1828, en el seno Almirantazgo, Tierra del Fuego, Chile. Anales del Instituto de la Patagonia, Chile 31: 91-96.

Anderson ORJ, CJ Small, JP Croxall, EK Dunn, B Sullivan, $O$ Yates, A Black. 2011. Global seabird bycatch in longline fisheries. Endangered Species Research 14: 91-106.
Arata J \& JC Xavier. 2003. The diet of black-browed albatrosses at the Diego Ramirez islands, Chile. Polar Biology 26: 638-647.

Aravena JC \& BH Luckman. 2009. Spatio-temporal rainfall patterns in Southern South America. International Journal of Climatology 29: 2106-2120.

Arntz WE, M Gorny, R Soto, MA Lardies, M Retamal \& I Wehrtmann. 1999. Species composition and distribution of decapod crustaceans in the waters off Patagonia and Tierra del Fuego, South America. Scientia Marina 63(Supl.1): 303-314.

Beuchel F \& B Gulliksen. 2008. Temporal patterns of benthic community development in an Arctic fjord (Kongsfjorden, Svalbard): results of a 24-year manipulation study. Polar Biology 31: 913-924.

Bibby C, N Burgess, D Hill \& S Mustoe. 2000. Birds census techniques, 302 pp. Academic Press, London.

Bingham M \& TM Hermann. 2008. Magellanic penguin (Spheniscidae) monitoring results for Magdalena Island (Chile) 2000-2008. Anales del Instituto de la Patagonia, Chile 36: 19-32.

Brown RGB, F Cooke, PK Kinnear \& EL Mills. 1975. Summer seabird distributions in Drake Passage, the Chilean Fjords and off southern South America. Ibis 117: 339-356.

Clarke KR \& RN Gorley. 2001. Primer v5: User manual/ tutorial, 91 pp. PRIMER- E, Plymouth.

Clarke KR \& RM Warwick. 2001. Change in marine communities: an approach to statistical analysis and interpretation, 172 pp. PRIMER-E, Plymouth.

Creuwels JCS, S Poncet, PJ Hodum \& JA Van Franeker. 2007. Distribution and abundance of the Southern Fulmar Fulmarus glacialoides. Polar Biology 30: 1083-1097.

Cursach JA, JR Rau \& CN Tobar. 2010a. Aves en un humedal marino del sur de Chile. Revista de Biología Marina y Oceanografía 45: 441-450.

Cursach JA, A Simeone, R Matus, O Soto, R Schlatter, C Tobar \& J Ojeda. 2010b. Distribución reproductiva del cormorán imperial (Phalacrocorax atriceps) en Chile. Boletín Chileno de Ornitología, Chile 16: 9-16.

Ferrari S, B Alegre \& P Gandini. 2004. Dieta del cormorán imperial (Phalacrocorax atriceps) en el sur de Santa Cruz (Patagonia, Argentina). Revista Ornitología Neotropical 15: 103-110.

Fraser PA. 2009. Westland petrels (Procellaria westlandica) off the coast of Chile. Notornis, Nueva Zelanda 56: 98-99.

García GO \& A Gómez-Laich. 2007. Abundancia y riqueza específica en un ensamble de aves marinas y costeras del sudeste de la provincia de Buenos Aires, Argentina. Hornero 22: 9-16.

González-Zevallos D \& P Yorio. 2006. Seabird use of discards and incidental captures at the Argentine hake trawl fishery in the Golfo San Jorge, Argentina. Marine Ecology Progress Series 316: 175-183.

Hudson AV \& RW Furness. 1988. The behaviour of seabirds foraging at fishing boats around Shetland. Ibis 131: 225237. 
Imberti S. 2005. Distribución otoñal de aves marinas y terrestres en los canales chilenos. Anales del Instituto de la Patagonia, Chile 33: 21-30.

Jehl JR. 1973. The distribution of marine birds in Chilean waters in Winter. Auk 90: 114-135.

Johnsgard PA. 1993. Cormorants, darters, and pelicans of the world, 445 pp. Smithsonian Institution Press, Washington.

Mackley EK, RA Phillips, JRD Silk, ED Wakefield, V Afanasyev, JW Fox \& RW Furness. 2010. Free as a bird? Activity patterns of albatrosses during the nonbreeding period. Marine Ecology Progress Series 406: 291-303.

Montevecchi WA. 2002. Interactions between fisheries and seabirds. En: Schreiber EA \& J Burger (eds). Biology of marine birds, 722 pp. CRC Press, Washington.

Moreno CA \& G Robertson. 2008. ¿Cuántos albatros de ceja negra, Thalassarche melanophrys (Temminck, 1828) anidan en Chile? Anales del Instituto de la Patagonia, Chile 36: 89-91.

Moreno CA, PS Rubilar, E Marschoff \& L Benzaquen. 1996. Factors affecting the incidental mortality of seabirds in the Dissostichus eleginoides fishery in the southwest Atlantic (subarea 48.3, 1995 season). CCAMLR Science 3: 79-91.

Moreno CA, JA Arata, P Rubilar, R Hucke-Gaete \& G Robertson. 2006. Artisanal longline fisheries in Southern Chile: Lessons to be learned to avoid incidental seabird mortality. Biological Conservation 127: 27-36.

Moreno CA, R Castro, LJ Mújica \& P Reyes. 2008. Significant conservation benefits obtained from the use of a new fishing gear in the Chilean Industrial Patagonian Toothfish Fishery. CCAMLR Science 15: 79-91.

Moreno CE. 2001. Métodos para medir la biodiversidad. M\&TManuales y Tesis SEA 1: 1-84. Sociedad Entomológica Aragonesa, Zaragoza.

Orta J. 1992. Family Phalacrocoracidae (Cormorants). In: Del Hoyo J, A Elliot \& J Sargatal (eds). Handbook of the birds of the word 1: 326-353. Lynx Ediciones, Barcelona.

Pennycuick CJ. 1982. The flight of petrels and albatrosses (Procellariiformes), observed in South Georgia and its vicinity. Philosophical Transactions of the Royal Society of London, Series B 300: 75-106.

Phillips RA, JP Croxall, JRD Silk \& DR Briggs. 2008. Foraging ecology of albatrosses and petrels from South Georgia: two decades of insights from tracking technologies. Aquatic Conservation: Marine and Freshwater Ecosystems 17: S6-S21.

Santana A, C Porter, N Butorovic \& C Olave. 2006. Primeros antecedentes climatológicos de estaciones automáticas (AWS) en el canal Beagle, Magallanes, Chile. Anales del Instituto de la Patagonia, Chile 34: 5-20.

Schlatter R \& A Simeone. 1999. Estado del conocimiento y conservación de las aves en mares chilenos. Estudios Oceanológicos 18: 25-33.
Silva N \& C Calvete. 2002. Características oceanográficas físicas y químicas de canales australes chilenos entre el Golfo de Penas y el Estrecho de Magallanes (Crucero CIMAR-FIORDOS 2). Ciencia y Tecnología del Mar 25: 23-88.

Simpson EH. 1949. Measurement of diversity. Nature 163: 688.

Soberón J \& J Llorente. 1993. The use of the species accumulation functions for the prediction of species richness. Conservation Biology 7: 480-488.

Suazo CG. 2008. Black-browed albatross foraging on jellyfish prey in the southeast Pacific coast, southern Chile. Polar Biology 31: 755-757.

Tasker ML, PH Jones, T Dixon, BF Blake. 1984. Counting seabirds at sea from ships: A review of methods employed and a suggestion for a standardized approach. The Auk 101: 567- 577.

Tasker ML, CJ Camphuysen, J Cooper, S Garthe, WA Montevecchi \& SJM Blaber. 2000. The impacts of fishing on marine birds. ICES Journal of Marine Science 57: 531547.

Tickell WLN. 2000. Albatrosses, 448 pp. Yale University Press, London.

Tickell WLN \& R Pinder. 1975. Breeding biology of the blackbrowed albatross Diomedea melanophris and grey-headed albatross D. chrysostoma at Bird Island, South Georgia. Ibis 117: 433-451.

Venegas C. 1999. Estado de conservación de las especies de pingüinos en la región de Magallanes, Chile. Estudios Oceanológicos 18: 45-56.

Wakefield ED, RA Phillips, PN Trathan, J Arata, R Gales, N Huin, G Robertson, SM Waugh, H Weimerskirch \& J Matthiopoulos. 2011. Habitat preference, accessibility, and competition limit the global distribution of breeding Black-browed Albatrosses. Ecological Monographs. 81: 141-167.

Weidinger K. 1997. Breeding cycle of the Cape petrel Daption capense at Nelson Island, Antarctica. Polar Biology 17: 469-472.

Weimerskirch H, D Capdeville \& G Duhamel. 2000. Factors affecting the number and mortality of seabirds attending trawlers and long-liners in the Kerguelen area. Polar Biology 23: $236-249$

Williams TD. 1995. The penguins, 295 pp. Oxford University Press, Oxford.

Wilson RP, JA Scolaro, D Grémillet, M Kierspel, S Laurenti, J Upton, H Gallelli, F Quitana, E Frere, G Müller, MT Straten \& I Zimmer. 2005. How do Magellanic Penguins cope with variability in their access to prey? Ecological Monographs 75: 379-401. 